RIMS-99?

hep-th/9501nnn

Jan 1995

\title{
Annihilation poles of a Smirnov-type integral formula for solutions to quantum Knizhnik-Zamolodchikov equation
}

\author{
Takeo Kojima ${ }^{1}$, Kei Miki ${ }^{2}$ and Yas-Hiro Quano ${ }^{3 *}$ \\ ${ }^{1}$ Research Institute for Mathematical Sciences, Kyoto University, Kyoto 606-01, Japan \\ ${ }^{2}$ Department of Mathematical Sciences, Faculty of Engineering Sciences, Osaka University, Toyonaka, \\ Osaka 560, Japan \\ ${ }^{3}$ Department of Mathematics, University of Melbourne, Parkville, Victoria 3052, Australia
}

\begin{abstract}
$\underline{\text { ABSTRACT }}$
We consider the recently obtained integral representation of quantum Knizhnik-Zamolodchikov equation of level 0 . We obtain the condition for the integral kernel such that these solutions satisfy three axioms for form factor á la Smirnov. We discuss the relation between this integral representation and the form factor of XXZ spin chain.
\end{abstract}

\footnotetext{
^ Supported by the Australian Research Council.
} 


\section{Introduction}

In [1] an integral formula of the Smirnov type was presented to the quantum Knizhnik-Zamolodchikov ( $q$ $\mathrm{KZ}$ ) equation [2] of level 0 associated with the vector representation of the quantum affine algebra $U_{q}\left(\widehat{\mathfrak{s l}}_{2}\right)$. The $U_{q}\left(\widehat{\mathfrak{s l}_{n}}\right)$ generalization was studied in [3]. In these formulae, the freedom of solutions to $q$-KZ equation corresponds to the choice of integral kernel with the cycle of integration being fixed. The present paper is a step towards the determination of the integral kernel given in [1] by studying the annihilation pole structures of the solutions.

In the pioneering works 团, Smirnov constructed the integral formulae of form factors of the sine-Gordon model that satisfy three axioms: (i) $S$ matrix symmetry; (ii) (deformed) cyclicity; (iii) annihilation pole condition. He utilized these axioms to construct the matrix elements of local operators. Refs [1, 3] were based on Smirnov's observation [5] that (i) and (ii) imply the $q$-KZ equation of level 0 . In these works, instead of solving the $q$-KZ equation directly, a system of difference equations arising from (i') the $R$ matrix symmetry and (ii) the deformed cyclicity were considered. At this moment the integral kernel of the formula is arbitrary except that it satisfies appropriate symmetries and quasi-periodicity conditions. These results can be easily modified so as to enjoy (i) instead of (i') for the $S$ matrix having the crossing symmetry. In this paper we shall

derive the condition for the integral kernel such that these solutions satisfy the third axiom for the $U_{q}\left(\widehat{\mathfrak{s l}}_{2}\right)$ case.

The $q$-KZ equation was originally introduced by Frenkel and Reshetikhin [2] and was found to be the master equation for the form factors of solvable lattice models with the quantum affine symmetries [6, 7]. In this approach, the form factors can be calculated by utilizing the vertex operators of the algebra. (See [8, 9 , for explicit calculations.) Moreover, the form factors of appropriate operators were shown to satisfy Smirnov's three axioms [1, 10, 11. Since our $S$ matrix coincides with the one appearing in the XXZ model, our solutions are expected to be related to the form factors of some operators in the model.

The present paper is organized as follows. In section 2 we formulate the problem and summarize our result. In sections 3 and 4 we prove our result. In section 5 we discuss our solutions in the context of the $X X Z$ model.

\section{Problem and Result.}

The purpose of this section is to formulate the problem, thereby fixing our notations, and to state our result.

For a fixed complex parameter $q$ such that $0<|q|<1$, let $U=U_{q}^{\prime}\left(\widehat{\mathfrak{s l}}_{2}\right)$ be a $\mathbb{C}$ algebra generated by $e_{i}$, $f_{i}$ and $t_{i}(i=0,1)$ that satisfy

$$
\left[e_{i}, f_{j}\right]=\delta_{i j} \frac{t_{i}-t_{i}^{-1}}{q-q^{-1}}, \quad t_{i} e_{j} t_{i}^{-1}=q^{4 \delta_{i j}-2} e_{j}, \quad t_{i} f_{j} t_{i}^{-1}=q^{2-4 \delta_{i j}} f_{j}, \quad t_{i} t_{j}=t_{j} t_{i}, \quad t_{i} t_{i}^{-1}=t_{i}^{-1} t_{i}=1
$$

and the serre relations 12. Let $\Delta$ be the following coproduct of $U$

$$
\Delta\left(e_{i}\right)=e_{i} \otimes 1+t_{i} \otimes e_{i}, \quad \Delta\left(f_{i}\right)=f_{i} \otimes t_{i}^{-1}+1 \otimes f_{i}, \quad \Delta\left(t_{i}\right)=t_{i} \otimes t_{i},
$$

and set $\Delta^{\prime}=\sigma \circ \Delta$, where $\sigma(x \otimes y)=y \otimes x$ for $x, y \in U$. Set $V \cong \mathbb{C} v_{+} \oplus \mathbb{C} v_{-}$and let $\left(\pi_{\zeta}, V\right)(\zeta \in \mathbb{C} \backslash\{0\})$ 
signify the vector representation of $U$ defined by

$$
\begin{array}{lll}
\pi_{\zeta}\left(e_{1}\right)\left(v_{+}, v_{-}\right)=\zeta\left(0, v_{+}\right), & \pi_{\zeta}\left(f_{1}\right)\left(v_{+}, v_{-}\right)=\zeta^{-1}\left(v_{-}, 0\right), & \pi_{\zeta}\left(t_{1}\right)\left(v_{+}, v_{-}\right)=\left(q v_{+}, q^{-1} v_{-}\right) \\
\pi_{\zeta}\left(e_{0}\right)\left(v_{+}, v_{-}\right)=\zeta\left(v_{-}, 0\right), & \pi_{\zeta}\left(f_{0}\right)\left(v_{+}, v_{-}\right)=\zeta^{-1}\left(0, v_{+}\right), & \pi_{\zeta}\left(t_{0}\right)\left(v_{+}, v_{-}\right)=\left(q^{-1} v_{+}, q v_{-}\right) .
\end{array}
$$

We shall later use the following abbreviation for its tensor product representation via $\Delta$

$$
\pi_{\left(\zeta_{1}, \cdots, \zeta_{N}\right)}(y)=\left(\pi_{\zeta_{1}} \otimes \cdots \otimes \pi_{\zeta_{N}}\right) \circ \Delta^{(N-1)}(y), \quad y \in U
$$

Let $R(\zeta) \in \operatorname{End}(V \otimes V)$ be the $R$ matrix of the six vertex model

$$
R(\zeta) v_{\varepsilon_{1}^{\prime}} \otimes v_{\varepsilon_{2}^{\prime}}=\sum_{\varepsilon_{1}, \varepsilon_{2}} v_{\varepsilon_{1}} \otimes v_{\varepsilon_{2}} R(\zeta)_{\varepsilon_{1}^{\prime} \varepsilon_{2}^{\prime}}^{\varepsilon_{1} \varepsilon_{2}}
$$

where the nonzero entries are given by

$$
\begin{aligned}
& R(\zeta)_{++}^{++}=R(\zeta)_{--}^{--}=1, \\
& R(\zeta)_{+-}^{+-}=R(\zeta)_{-+}^{-+}=b(\zeta)=\frac{\left(1-\zeta^{2}\right) q}{1-\zeta^{2} q^{2}}, \\
& R(\zeta)_{-+}^{+-}=R(\zeta)_{+-}^{-+}=c(\zeta)=\frac{\left(1-q^{2}\right) \zeta}{1-\zeta^{2} q^{2}} .
\end{aligned}
$$

Then the following intertwining property holds [12]:

$$
R\left(\zeta_{1} / \zeta_{2}\right)\left(\pi_{\zeta_{1}} \otimes \pi_{\zeta_{2}}\right) \circ \Delta(y)=\left(\pi_{\zeta_{1}} \otimes \pi_{\zeta_{2}}\right) \circ \Delta^{\prime}(y) R\left(\zeta_{1} / \zeta_{2}\right) .
$$

We further introduce the scattering matrix $S$ [6, 7]

$$
S(\zeta)=S_{0}(\zeta) R(\zeta)
$$

where

$$
S_{0}(\zeta)=\zeta \frac{\left(z^{-1} ; q^{4}\right)_{\infty}\left(z q^{2} ; q^{4}\right)_{\infty}}{\left(z ; q^{4}\right)_{\infty}\left(z^{-1} q^{2} ; q^{4}\right)_{\infty}}, \quad\left(a ; p_{1}, \cdots, p_{n}\right)_{\infty}=\prod_{k_{i} \geq 0}\left(1-a p_{1}^{k_{1}} \cdots p_{n}^{k_{n}}\right)
$$

and $z=\zeta^{2}$. In what follows we shall work with the tensor products of the vector space $V$ 's. Following the usual convention, for $M \in \operatorname{End}(\mathrm{V})$ we let $M_{j}$ denote the operator on $V^{\otimes N}$ acting as $M$ on the $j$-th tensor component and as identity on the other components. Similarly for $X=S$ or $R$, we let $X_{j k}(\zeta)$ $(j \neq k)$ signify the operator on $V^{\otimes N}$ acting as $X(\zeta)$ on the $(j, k)$-th tensor components and as identity on the other components. In particular we have $X_{k j}(\zeta)=P_{j k} X_{j k}(\zeta) P_{j k}$, where $P \in \operatorname{End}(V \otimes V)$ stands for the transposition $P(x \otimes y)=y \otimes x$. We often use the following abbreviations

$$
\begin{aligned}
& X_{1, \cdots, N \mid N+1}\left(\zeta_{1}, \cdots, \zeta_{N} \mid \zeta_{N+1}\right)=X_{1, N+1}\left(\zeta_{1} / \zeta_{N+1}\right) \cdots X_{N, N+1}\left(\zeta_{N} / \zeta_{N+1}\right), \\
& X_{N+1 \mid 1, \cdots, N}\left(\zeta_{N+1} \mid \zeta_{1}, \cdots, \zeta_{N}\right)=X_{N+1, N}\left(\zeta_{N+1} / \zeta_{N}\right) \cdots X_{N+1,1}\left(\zeta_{N+1} / \zeta_{1}\right),
\end{aligned}
$$

where $X=S$ or $R$.

The main properties of $S(\zeta)$ are the Yang-Baxter equation

$$
S_{12}\left(\zeta_{1} / \zeta_{2}\right) S_{13}\left(\zeta_{1} / \zeta_{3}\right) S_{23}\left(\zeta_{2} / \zeta_{3}\right)=S_{23}\left(\zeta_{2} / \zeta_{3}\right) S_{13}\left(\zeta_{1} / \zeta_{3}\right) S_{12}\left(\zeta_{1} / \zeta_{2}\right),
$$

the initial condition

$$
S(1)=-P,
$$


the unitarity relation

$$
S_{12}\left(\zeta_{1} / \zeta_{2}\right) S_{21}\left(\zeta_{2} / \zeta_{1}\right)=1
$$

and the crossing symmetry

$$
S_{12}(\zeta) v_{\varepsilon} \otimes u_{\sigma}=\sigma S_{31}(-\sigma / q \zeta) v_{\varepsilon} \otimes u_{\sigma}
$$

where $u_{\sigma}=v_{-} \otimes v_{+}+\sigma v_{+} \otimes v_{-} \quad(\sigma= \pm)$.

Let $V^{(n l)}$ be the subspace of $V^{\otimes N}$ defined by

$$
V^{(n l)}=\oplus \mathbb{C} v_{\varepsilon_{1}} \otimes \cdots \otimes v_{\varepsilon_{N}}
$$

where the sum is taken over $\varepsilon_{j}= \pm 1$ with fixed

$$
n=\sharp\left\{j \mid \varepsilon_{j}=-\right\}, \quad l=\sharp\left\{j \mid \varepsilon_{j}=+\right\} \quad(n+l=N)
$$

and let us consider a $V^{(n l)}$-valued function

$$
G_{\varepsilon}^{(n l)}\left(\zeta_{1}, \cdots, \zeta_{N}\right)=\sum v_{\varepsilon_{1}} \otimes \cdots \otimes v_{\varepsilon_{N}} G_{\varepsilon}^{(n l)}\left(\zeta_{1}, \cdots, \zeta_{N}\right)^{\varepsilon_{1} \cdots \varepsilon_{N}} \quad(\varepsilon= \pm)
$$

Our problem is to obtain the function family $G_{\varepsilon}^{(n l)}\left(\zeta_{1}, \cdots, \zeta_{N}\right)(\varepsilon= \pm, n, l=1,2, \cdots)$ that satisfy the following three axioms,

\section{1. $S$ matrix symmetry}

$$
P_{j j+1} G_{\varepsilon}^{(n l)}\left(\cdots, \zeta_{j+1}, \zeta_{j}, \cdots\right) \quad=S_{j j+1}\left(\zeta_{j} / \zeta_{j+1}\right) G_{\varepsilon}^{(n l)}\left(\cdots, \zeta_{j}, \zeta_{j+1}, \cdots\right) \quad(1 \leq j \leq N-1) .
$$

\section{Deformed cyclicity}

$$
P_{12} \cdots P_{N-1 N} G_{\varepsilon}^{(n l)}\left(\zeta_{2}, \cdots, \zeta_{N}, \zeta_{1} q^{-2}\right)=r_{\varepsilon}^{(l-n)}\left(\zeta_{1}\right) D_{1}^{(l-n)} G_{\varepsilon}^{(n l)}\left(\zeta_{1}, \cdots, \zeta_{N}\right) .
$$

3. Annihilation pole condition The $G_{\varepsilon}^{(n l)}(\zeta)$ has poles at $\zeta_{N}=-\sigma \zeta_{N-1} / q(\sigma= \pm)$ and the residue is given by

$$
\begin{aligned}
& \operatorname{Res}_{\zeta_{N} /\left(-\sigma \zeta_{N-1} q^{-1}\right)=1} G_{\varepsilon}^{(n l)}(\zeta) \\
= & \frac{1}{2}\left(I-\sigma^{N+1} r_{\varepsilon}^{(l-n)}\left(-\sigma \zeta_{N-1} q\right) D_{N}^{(l-n)} S_{N-1 \mid 1, \cdots, N-2}\left(\zeta_{N-1} \mid \zeta^{\prime}\right)\right) G_{\sigma \varepsilon}^{(n-1 l-1)}\left(\zeta^{\prime}\right) \otimes u_{\sigma} .
\end{aligned}
$$

Here $r_{\varepsilon}^{(k)}(\zeta)=\varepsilon r^{(k)}(\zeta), r^{(k)}(\zeta)$ are scalar functions satisfying

$$
r^{(k)}(\zeta) r^{(k)}(-\sigma \zeta q)=\sigma^{N}
$$

$D^{(k)}=\operatorname{diag}\left(\delta^{(k)}, \delta^{(k)-1}\right)$, and $I$ is the identity operator. In this paper we employ the convention $\operatorname{Res}_{x / y=1} G(x)=$ $F(y)$ when $G(x)=\frac{1}{x / y-1} F(y)+O(1)$ at $x \simeq y$, and we often use the abbreviations $(\zeta)=\left(\zeta_{1}, \cdots, \zeta_{N}\right)$ and $\left(\zeta^{\prime}\right)=\left(\zeta_{1}, \cdots, \zeta_{N-2}\right)$. In section 5 , we shall discuss the physical meaning of the above axioms and the reason why we introduce $r^{(k)}(\zeta)$.

\section{Remark}

1. The action of the scattering matrix $S(\zeta)$ preserves the values of $n$ and $l$. The $S$ matrix further enjoys the $\mathbb{Z}_{2}$-symmetry. Therefore we can consider $G_{\varepsilon}^{(n l)}(\zeta) \in V^{(n l)}$ and may assume $n \leq l$ without loss of generality. 
2. The condition for $r^{(k)}(\zeta)$ (2.10) follows from the consistency of the three axioms. This can be seen from (4.3) and the argument below it. Similarly we can show that the diagonal operator $r_{\varepsilon}^{(k)}(\zeta) D^{(k)}$ depends only on $l-n$.

Hereafter we shall restrict ourselves to the case $\delta^{(k)}=q^{-k / 2}$ and $n \leq l$, since for this choice the solutions to the first two axioms were already obtained in 迆. In this paper, we shall derive the condition for these solutions to satisfy the third axiom. Before we state our result, we shall explain how the solutions to the first two axioms are obtained from the results of [1]. Set

$$
G_{\varepsilon}^{(n l)}(\zeta)=\bar{G}_{\varepsilon}^{(n l)}(\zeta) \prod_{1 \leq i<j \leq N} g\left(z_{i} / z_{j}\right) \prod_{j=1}^{N} \zeta_{j}^{j-N}
$$

where

$$
g(z)=\frac{\left(z ; q^{4}, q^{4}\right)_{\infty}\left(q^{4} / z ; q^{4}, q^{4}\right)_{\infty}}{\left(q^{2} z ; q^{4}, q^{4}\right)_{\infty}\left(q^{6} / z ; q^{4}, q^{4}\right)_{\infty}}
$$

Then the first two axioms can be recast as follows:

$$
\begin{gathered}
P_{j j+1} \bar{G}_{\varepsilon}^{(n l)}\left(\cdots, \zeta_{j+1}, \zeta_{j}, \cdots\right)=R_{j j+1}\left(\zeta_{j} / \zeta_{j+1}\right) \bar{G}_{\varepsilon}^{(n l)}\left(\cdots, \zeta_{j}, \zeta_{j+1}, \cdots\right), \\
P_{12} \cdots P_{N-1 N} \bar{G}_{\varepsilon}^{(n l)}\left(\zeta_{2}, \cdots, \zeta_{N}, \zeta_{1} q^{-2}\right)=r_{\varepsilon}\left(\zeta_{1}\right) D_{1}^{(l-n)} \bar{G}_{\varepsilon}^{(n l)}\left(\zeta_{1}, \cdots, \zeta_{N}\right) \prod_{j=2}^{N} \frac{\zeta_{j}}{\zeta_{1}}
\end{gathered}
$$

Therefore, though the deformed cyclicity (2.13) is different from the one in [1] by a multiplication factor, $\bar{G}_{\varepsilon}^{(n l)}(\zeta)$ is similarly shown to have the following integral formula

$$
\bar{G}_{\varepsilon}^{(n l)}(\zeta)=\frac{1}{m !} \prod_{\mu=1}^{m} \oint_{C^{(N)}} \frac{d x_{\mu}}{2 \pi i} \Psi_{\varepsilon}^{(n l)}\left(x_{1}, \cdots, x_{m} \mid \zeta_{1}, \cdots, \zeta_{N}\right)\left\langle\Delta^{(n l)}\right\rangle\left(x_{1}, \cdots, x_{m} \mid \zeta_{1}, \cdots, \zeta_{N}\right) .
$$

Here $m=n-1$ for $n=l$ and $m=n$ for $n<l$, and $\Delta^{(n l)}\left(x_{1}, \cdots, x_{m}\left|z_{1}, \cdots, z_{n}\right| z_{n+1}, \cdots, z_{N}\right)$ is the same polynomial obtained in [1]. (See (3.7) below, and 1] for further details.) $\left\langle\Delta^{(n l)}\right\rangle\left(x_{1}, \cdots, x_{m} \mid \zeta_{1}, \cdots, \zeta_{N}\right) \in V^{(n l)}$ is the vector defined from this polynomial as follows

$$
\begin{aligned}
\left\langle\Delta^{(n l)}\right\rangle\left(x_{1}, \cdots, x_{m} \mid \zeta_{1}, \cdots, \zeta_{N}\right)^{-\cdots-+\cdots+} & =\frac{\Delta^{(n l)}\left(x_{1}, \cdots, x_{m}\left|z_{1}, \cdots, z_{n}\right| z_{n+1}, \cdots, z_{N}\right)}{\prod_{j=1}^{n} \prod_{i=n+1}^{N}\left(z_{i}-z_{j} \tau^{2}\right)} \prod_{j=1}^{n} \zeta_{j}, \\
P_{j j+1}\left\langle\Delta^{(n l)}\right\rangle\left(x_{1}, \cdots, x_{m} \mid \cdots, \zeta_{j+1}, \zeta_{j}, \cdots\right) & =R_{j j+1}\left(\zeta_{j} / \zeta_{j+1}\right)\left\langle\Delta^{(n l)}\right\rangle\left(x_{1}, \cdots, x_{m} \mid \cdots, \zeta_{j}, \zeta_{j+1}, \cdots\right),
\end{aligned}
$$

where and hereafter $\tau=q^{-1}$. The integration $\oint_{C^{(N)}} d x_{\mu}$ is along a simple closed curve $C^{(N)}=C^{(N)}\left(z_{1}, \cdots, z_{N}\right)$ oriented anti-clockwise, which encircles the points $z_{j} \tau^{-1-4 k}(1 \leq j \leq N, k \geq 0)$ but not $z_{j} \tau^{1+4 k}(1 \leq j \leq N, k \geq$ $0)$. The kernel $\Psi_{\varepsilon}^{(n l)}$ has the form

$$
\Psi_{\varepsilon}^{(n l)}\left(x_{1}, \cdots, x_{m} \mid \zeta_{1}, \cdots, \zeta_{N}\right)=\vartheta_{\varepsilon}^{(n l)}\left(x_{1}, \cdots, x_{m} \mid \zeta_{1}, \cdots, \zeta_{N}\right) \prod_{\mu=1}^{m} \prod_{j=1}^{N} \psi\left(\frac{x_{\mu}}{z_{j}}\right) .
$$

Here

$$
\psi(z)=\frac{1}{\left(z q ; q^{4}\right)_{\infty}\left(z^{-1} q ; q^{4}\right)_{\infty}}
$$

and $\vartheta_{\varepsilon}^{(n l)}$ is an arbitrary function that has the following properties: 
- it is anti-symmetric and holomorphic in the $x_{\mu} \in \mathbb{C} \backslash\{0\}$,

- it is symmetric and meromorphic in the $\zeta_{j} \in \mathbb{C} \backslash\{0\}$,

- it has the two transformation properties

$$
\begin{gathered}
\frac{\vartheta_{\varepsilon}^{(n l)}\left(x_{1}, \cdots, x_{m} \mid \zeta_{1}, \cdots, \zeta_{j} \tau^{2}, \cdots, \zeta_{N}\right)}{\vartheta_{\varepsilon}^{(n l)}\left(x_{1}, \cdots, x_{m} \mid \zeta_{1}, \cdots, \zeta_{N}\right)}=\tau^{N / 2} r_{\varepsilon}^{(l-n)}\left(\zeta_{j}\right) \prod_{\mu=1}^{m} \frac{-z_{j} \tau}{x_{\mu}} \prod_{\substack{k=1 \\
k \neq j}}^{N} \frac{\zeta_{k}}{\zeta_{j}}, \\
\frac{\vartheta_{\varepsilon}^{(n l)}\left(x_{1}, \cdots, x_{\mu} \tau^{4}, \cdots, x_{m} \mid \zeta_{1}, \cdots, \zeta_{N}\right)}{\vartheta_{\varepsilon}^{(n l)}\left(x_{1}, \cdots, x_{m} \mid \zeta_{1}, \cdots, \zeta_{N}\right)}=\prod_{j=1}^{N} \frac{-x_{\mu} \tau}{z_{j}} .
\end{gathered}
$$

Note that the first transformation function (2.15) takes a form different from the one in [1] because of the modification of (2.13).

Now we are in a position to state the main result of this paper:

Theorem 2.1 The function family $G_{\varepsilon}^{(n l)}(\zeta)$ defined in (2.11) and (2.14) satisfies the annihilation pole condition (2.9) if $\vartheta_{\varepsilon}^{(n l)}$ satisfies the following recurrence relation

$$
\frac{\vartheta_{\varepsilon}^{(n l)}\left(x_{1}, \cdots, x_{m-1}, z_{N-1} \tau \mid \zeta_{1}, \cdots, \zeta_{N-2}, \zeta_{N-1},-\sigma \zeta_{N-1} \tau^{-1}\right)}{\vartheta_{\sigma \varepsilon}^{(n-1, l-1)}\left(x_{1}, \cdots, x_{m-1} \mid \zeta_{1}, \cdots, \zeta_{N-2}\right)}=c^{(n l)} z_{N-1}^{N-m-1} \prod_{\mu=1}^{m-1} \theta\left(q x_{\mu} / z_{N-1} \mid q^{2}\right) .
$$

where

$$
\begin{aligned}
& \theta(z \mid q)=(z ; q)_{\infty}(q / z ; q)_{\infty}, \\
& c^{(n l)}=(-\tau)^{m-n-2 l+3}\left(q^{2} ; q^{2}\right)_{\infty}^{2} \frac{\left(q^{4} ; q^{4}, q^{4}\right)_{\infty}^{2}}{\left(q^{2} ; q^{4}, q^{4}\right)_{\infty}^{2}}
\end{aligned}
$$

There exists a nontrivial example of the function $\vartheta_{\varepsilon}^{(n l)}$ which satisfies the above conditions for a particular choice of $r^{(k)}(\zeta)$. See section 5 . We shall check the $(-\cdots-+\cdots+)$-component of the annihilation pole condition $(2.9)$ in the next section and show that Theorem 2.1 follows from it in section 4 .

\section{The Annihilation Pole Condition for the Extreme Component}

In this section we show the $(-\cdots-+\cdots+)$-component of the annihilation pole condition (2.9). In terms of $\bar{G}_{\varepsilon}^{(n l)}$, it can be recast as

Proposition 3.1 If $\vartheta_{\varepsilon}^{(n l)}$ satisfies (2.17), then the following holds

$$
\begin{aligned}
& -2 \frac{\left(q^{2} ; q^{4}, q^{4}\right)_{\infty}^{2}}{\left(q^{4} ; q^{4}, q^{4}\right)_{\infty}^{2}} \frac{\sigma^{N+1} \tau^{(n-l) / 2} \operatorname{Res}_{\zeta_{N} /\left(-\sigma \zeta_{N-1} \tau\right)=1} \bar{G}_{\varepsilon}^{(n l)}(\zeta)^{-\cdots-+\cdots+\cdots+}\left(-\sigma \zeta_{N-1} q\right) \zeta_{N-1} \prod_{j=1}^{N-2}\left(\zeta_{j} \zeta_{N-1} \psi\left(\frac{\tau z_{N-1}}{z_{j}}\right)\right)}{=\left(R_{N-1 \mid 1, \cdots, N-2}\left(\zeta_{N-1} \mid \zeta^{\prime}\right) \bar{G}_{\sigma \varepsilon}^{(n-1 l-1)} \otimes u_{\sigma}\right)^{-\cdots-\cdots+} .}
\end{aligned}
$$


Proof From the definition of the $R$ matrix, the RHS is equal to

$$
\sum_{k=1}^{n} c\left(\frac{\zeta_{N-1}}{\zeta_{k}}\right) \prod_{j=k+1}^{n} b\left(\frac{\zeta_{N-1}}{\zeta_{j}}\right) \bar{G}_{\varepsilon}^{(n-1, l-1)}\left(\zeta^{\prime}\right)^{-\cdots \stackrel{k}{+} \cdots-\stackrel{n+1}{+} \cdots+} .
$$

Thanks to the $R$ symmetry (2.12), we have the following relation

$$
\bar{G}_{\varepsilon}^{(n-1, l-1)}\left(\zeta^{\prime}\right)^{-\cdots \stackrel{k}{+} \cdots-\stackrel{n+1}{+} \cdots+}=\prod_{j=k+1}^{n} \frac{1}{b}\left(\frac{\zeta_{k}}{\zeta_{j}}\right) \bar{G}_{\varepsilon, k}^{(n-1 l-1)}\left(\zeta^{\prime}\right)-\sum_{j=k+1}^{n} \frac{c}{b}\left(\frac{\zeta_{k}}{\zeta_{j}}\right) \prod_{\substack{i=k+1 \\ i \neq j}}^{n} \frac{1}{b}\left(\frac{\zeta_{j}}{\zeta_{i}}\right) \bar{G}_{\varepsilon, j}^{(n-1 l-1)}\left(\zeta^{\prime}\right)
$$

where

$$
\bar{G}_{\varepsilon, j}^{(n-1, l-1)}\left(\zeta^{\prime}\right)=\bar{G}_{\varepsilon}^{(n-1 l-1)}\left(\zeta_{1}, \stackrel{j}{\therefore}, \zeta_{n}, \zeta_{j}, \zeta_{n+1}, \cdots, \zeta_{N-2}\right)^{-\cdots-+\cdots+} \quad(1 \leq j \leq n) .
$$

Note that (3.2) contains only one term proportional to $\bar{G}_{\varepsilon, 1}^{(n-1 l-1)}\left(\zeta^{\prime}\right)$ when expressed in terms of $\bar{G}_{\varepsilon, j}^{(n-1 l-1)}\left(\zeta^{\prime}\right)$. Since the result should be symmetric with respect to $\zeta_{1}, \cdots, \zeta_{n}$, we obtain

$$
\mathrm{RHS}=\frac{\left(1-\tau^{2}\right) \zeta_{N-1}}{\prod_{j=1}^{n}\left(z_{N-1}-z_{j} \tau^{2}\right)} \sum_{k=1}^{n} \zeta_{k} \prod_{\substack{j=1 \\ j \neq k}}^{n} \frac{\left(z_{N-1}-z_{j}\right)\left(z_{k}-z_{j} \tau^{2}\right)}{z_{k}-z_{j}} \bar{G}_{\varepsilon, k}^{(n-1, l-1)}\left(\zeta^{\prime}\right)
$$

Let us turn to the LHS. In the calculation of the residue of

$$
\bar{G}_{\varepsilon}^{(n l)}(\zeta)^{-\cdots-+\cdots+}=\frac{1}{m !} \prod_{\mu=1}^{m} \oint_{C^{(N)}} \frac{d x_{\mu}}{2 \pi i} \Psi_{\varepsilon}^{(n l)}\left(x_{1}, \cdots, x_{m} \mid \zeta\right) \frac{\Delta^{(n l)}\left(x_{1}, \cdots, x_{m}\left|z_{1}, \cdots, z_{n}\right| z_{n+1}, \cdots, z_{N}\right)}{\prod_{j=1}^{n} \prod_{i=n+1}^{N}\left(z_{i}-z_{j} \tau^{2}\right)} \prod_{j=1}^{n} \zeta_{j},
$$

at $\zeta_{N}=-\sigma \zeta_{N-1} \tau$, we rewrite the integration as

$$
\prod_{\mu=1}^{m} \oint_{C^{(N)}} \frac{d x_{\mu}}{2 \pi i}=\prod_{\mu=1}^{m-1} \oint_{C^{\prime(N)}} \frac{d x_{\mu}}{2 \pi i}\left(\oint_{C^{\prime(N)}} \frac{d x_{m}}{2 \pi i}+m \operatorname{Res}_{x_{m}=z_{N} \tau^{-1}}\right)
$$

in order to avoid the pinch of the contour $C^{(N)}$. Here $C^{\prime(N)}$ is a simple anti-clockwise closed curve which encloses the same poles but $z_{N} \tau^{-1}$ as for $C^{(N)}$, and we used the symmetry of the integrand with respect to $x_{\mu}$ 's. Since the integrand is regular at $\zeta_{N}=-\sigma \zeta_{N-1} \tau$, only the second term of the RHS of (3.4) contributes to the residue. Note that $(2.17)$ is equivalent to

$$
\begin{aligned}
& \frac{\vartheta_{\varepsilon}^{(n l)}\left(x_{1}, \cdots, x_{m-1}, z_{N-1} \tau \mid \zeta^{\prime}, \zeta_{N-1},-\sigma \zeta_{N-1} \tau\right)}{\vartheta_{\sigma \varepsilon}^{(n-1, l-1)}\left(x_{1}, \cdots, x_{m-1} \mid \zeta^{\prime}\right)} \\
& =(-1)^{N-m+1} \tau^{3 N / 2-m-2} c^{(n l)} \sigma^{N+1} r_{\varepsilon}^{(l-n)}\left(-\sigma \zeta_{N-1} q\right) \prod_{j=1}^{N-2} \zeta_{j} \zeta_{N-1} \prod_{\mu=1}^{m-1} \frac{\theta\left(q x_{\mu} / z_{N-1} \mid q^{2}\right)}{x_{\mu}},
\end{aligned}
$$

from 2.15$)$. We thus find

$$
\begin{aligned}
\text { LHS } & =\frac{1}{(m-1) !} \prod_{\mu=1}^{m-1} \oint_{C^{\prime(N)}} \frac{d x_{\mu}}{2 \pi i} \Psi_{\sigma \varepsilon}^{(n-1 l-1)}\left(x_{1}, \cdots, x_{m-1} \mid \zeta^{\prime}\right) \prod_{j=1}^{n} \prod_{i=n+1}^{N-1} \frac{1}{z_{i}-z_{j} \tau^{2}} \prod_{j=1}^{n} \zeta_{j} \\
& \times(-1)^{l} \tau^{2-N} \zeta_{N-1} \frac{\Delta^{(n l)}\left(x_{1}, \cdots, x_{m-1}, z_{N-1} \tau\left|z_{1}, \cdots, z_{n}\right| z_{n+1}, \cdots, z_{N-1}, z_{N-1} \tau^{2}\right)}{\prod_{j=1}^{n}\left(z_{N-1}-z_{j}\right) \prod_{\mu=1}^{m-1}\left(x_{\mu}-z_{N-1} \tau\right)} .
\end{aligned}
$$

In order to show the equality of $(3.3)$ and (3.5), we shall prove 
Proposition 3.2 The following holds:

$$
\begin{aligned}
& \frac{\Delta^{(n l)}\left(x_{1}, \cdots, x_{m-1}, w \tau\left|z_{1}, \cdots, z_{n}\right| z_{n+1}, \cdots, z_{N-2}, w, w \tau^{2}\right)}{\prod_{j=1}^{n}\left(w-z_{j}\right) \prod_{\mu=1}^{m-1}\left(x_{\mu}-w \tau\right)}=\tau^{n} \sum_{k=1}^{n} \prod_{\substack{j=1 \\
j \neq k}}^{n} \frac{w-z_{j}}{z_{k}-z_{j}} \\
& \times\left\{(-\tau)^{l-2}\left(1-\tau^{2}\right) \prod_{i=n+1}^{N-2}\left(z_{i}-z_{k} \tau^{2}\right) \Delta^{(n-1 l-1)}\left(x_{1}, \cdots, x_{m-1}\left|z_{1}, \stackrel{k}{\wedge}, z_{n}\right| z_{k}, z_{n+1}, \cdots, z_{N-2}\right)\right. \\
& \left.+\sum_{\nu=1}^{m-1}(-1)^{m+\nu} h^{(N-2)}\left(x_{\nu} \mid z_{1}, \cdots, z_{N-2}\right) \Delta^{\prime(m-2)}\left(x_{1}, \stackrel{\nu}{\wedge}, x_{m-1}\left|z_{k}\right| z_{1}, \stackrel{k}{\wedge}, z_{n} \mid z_{n+1}, \cdots, z_{N-2}\right)\right\},
\end{aligned}
$$

Here $h^{(N)}\left(x \mid z_{1}, \cdots, z_{N}\right)$ is the polynomial defined in 团] and

$$
\begin{aligned}
& \Delta^{\prime(m-2)}\left(x_{1}, \cdots, x_{m-2}\left|z_{1}\right| z_{2}, \cdots, z_{n} \mid z_{n+1}, \cdots, z_{N-2}\right) \\
= & \operatorname{det}\left(\left(A_{\lambda}^{(n-1 l-1)}\left(x_{\mu}\left|z^{\prime}\right| z^{\prime \prime}\right)\right)_{\substack{1 \leq \mu \leq m-2 \\
n-m+1 \leq \lambda \leq n-1}},\left(f_{\lambda}^{(n-1 l-1)}\left(z_{1} \tau\left|z^{\prime}\right| z^{\prime \prime}\right)\right)_{n-m+1 \leq \lambda \leq n-1}\right)
\end{aligned}
$$

where $z^{\prime}=\left(z_{2}, \cdots, z_{n}\right)$ and $z^{\prime \prime}=\left(z_{n+1}, \cdots, z_{N-2}, z_{1}\right)$.

Proof. Let us recall [1] that

$$
\Delta^{(n l)}\left(x_{1}, \cdots, x_{m}\left|z_{1}, \cdots, z_{n}\right| z_{n+1}, \cdots, z_{N}\right)=\operatorname{det}\left(A_{\lambda}^{(n l)}\left(x_{\mu}\left|z_{1}, \cdots, z_{n}\right| z_{n+1}, \cdots, z_{N}\right)\right) \substack{1 \leq \mu \leq m \\ n-m+1 \leq \lambda \leq n}_{\substack{1 \leq m+1 \\ n}}
$$

where

$$
\begin{aligned}
A_{\lambda}^{(n l)}\left(x\left|a_{1}, \cdots, a_{n}\right| b_{1}, \cdots, b_{l}\right)= & \prod_{j=1}^{n}\left(x-a_{j} \tau\right) f_{\lambda}^{(n l)}\left(x\left|a_{1}, \cdots, a_{n}\right| b_{1}, \cdots, b_{l}\right) \\
& +\tau^{2(l-n+\lambda-1)} \prod_{i=1}^{l}\left(x-b_{i} \tau^{-1}\right) g_{\lambda}^{(n)}\left(x \mid a_{1}, \cdots, a_{n}\right) .
\end{aligned}
$$

As for the definition of $f_{\lambda}^{(n l)}$ and $g_{\lambda}^{(n)}$, see [1]. From

$$
A_{\lambda}^{(n l)}\left(w \tau\left|z_{1}, \cdots, z_{n}\right| z_{n+1}, \cdots, z_{N-2}, w, w \tau^{2}\right)=\tau^{n} \prod_{j=1}^{n}\left(w-z_{j}\right) f_{\lambda}^{(n l)}\left(w \tau\left|z_{1}, \cdots, z_{n}\right| z_{n+1}, \cdots, z_{N-2}, w, w \tau^{2}\right),
$$

and the determinant structure of $\Delta^{(n l)}$, we find that the LHS of 3.6$)$ is a polynomial in $w$. From the following properties ((4.3) and (4.15) in [1])

$$
\begin{aligned}
& A_{\lambda}^{(n l)}\left(x\left|a_{1}, \cdots, a_{n}\right| b_{1}, \cdots, b_{l}\right) \text { is linear with respect to } b_{i} \text { 's, } \\
& f_{\lambda}^{(n l)}\left(w \tau\left|z_{1}, \cdots, z_{n}\right| z_{n+1}, \cdots, z_{N-2}, w, w \tau^{2}\right) \text { is independent of } w,
\end{aligned}
$$

we further find that its degree is equal to $m-1$. Thus the LHS can be determined from $n(>m-1)$ values at $w=z_{k}(1 \leq k \leq n)$. For example, when $w=z_{1}$, using the recurrence relations of $A_{\lambda}^{(n l)}$ and $f_{\lambda}^{(n l)}$ 歫, its value is found to be

$$
\tau^{n} \operatorname{det}\left(\left(A_{\lambda}^{(n-1 l-1)}\left(x_{\mu}\left|z^{\prime}\right| z^{\prime \prime}\right)\right)_{\substack{1 \leq \mu \leq m-1 \\ n-m+1 \leq \lambda \leq n}},\left(f_{\lambda}^{(n-1 l-1)}\left(z_{1} \tau\left|z^{\prime}\right| z^{\prime \prime}\right)\right)_{n-m+1 \leq \lambda \leq n}\right)
$$

where $z^{\prime}$ and $z^{\prime \prime}$ are the same abbreviations as used in the definition of $\Delta^{\prime(m-2)}$. Then noting

$$
f_{n}^{(n-1 l-1)}\left(z_{1} \tau\left|z^{\prime}\right| z^{\prime \prime}\right)=(-\tau)^{l-2}\left(1-\tau^{2}\right) \prod_{i=n+1}^{N-2}\left(z_{i}-z_{1} \tau^{2}\right)
$$


we obtain (3.6).

After the substitution of (3.6) into (3.5) we can replace $C^{\prime(N)}\left(z_{1}, \cdots, z_{N}\right)$ by $C^{(N-2)}\left(z_{1}, \cdots, z_{N-2}\right)$. Moreover, thanks to the same argument as given in the proof of Lemma 3.2 in [1], the terms proportional to $h^{(N-2)}\left(x_{\nu} \mid z_{1}, \cdots, z_{N-2}\right)$ vanish after the integration. Hence Proposition 3.1 is proved.

\section{Proof of the Theorem}

In this section we shall complete the proof of Theorem 2.1. Firstly we show

Proposition 4.1 If the function family $G_{\varepsilon}^{(n l)}\left(\zeta_{1}, \cdots, \zeta_{N}\right) \in V^{(n l)},(\varepsilon= \pm, n, l=1,2, \cdots)$ satisfies,

1. $S$ - matrix symmetry (2.7),

2. Deformed cyclicity (2.8),

3. The $(-\cdots-+\cdots+)-$ component of the annihilation pole condition (2.9),

4. $\pi_{\left(\zeta_{1}, \cdots, \zeta_{N}\right)}\left(f_{0}\right)\left(G_{\varepsilon}^{(n l)}\left(\zeta_{1}, \cdots, \zeta_{N}\right)\right)=0$,

then the function family $G_{\varepsilon}^{(n l)}\left(\zeta_{1}, \cdots, \zeta_{N}\right)$ satisfies the annihilation pole condition 2.9.).

Proof. In this proof, for $Y \in V^{\otimes N}$ we define $Y^{\left[\varepsilon_{1} \varepsilon_{2}\right]} \in V^{\otimes N-2}$ by

$$
Y=\sum_{\varepsilon_{1} \varepsilon_{2}} Y^{\left[\varepsilon_{1} \varepsilon_{2}\right]} \otimes v_{\varepsilon_{1}} \otimes v_{\varepsilon_{2}}
$$

and call it the $\left[\varepsilon_{1} \varepsilon_{2}\right]$ component of $Y$.

Let $A_{\varepsilon, \sigma}^{(n l)}\left(\zeta^{\prime} \mid \zeta_{N-1}\right)$ and $B_{\varepsilon, \sigma}^{(n l)}\left(\zeta^{\prime} \mid \zeta_{N-1}\right)$ denote the LHS and RHS of the annihilation pole condition (2.9), respectively, and set $K_{\varepsilon, \sigma}^{(n l)}\left(\zeta^{\prime} \mid \zeta_{N-1}\right)=A_{\varepsilon, \sigma}^{(n l)}\left(\zeta^{\prime} \mid \zeta_{N-1}\right)-B_{\varepsilon, \sigma}^{(n l)}\left(\zeta^{\prime} \mid \zeta_{N-1}\right)$. The aim of this proof is to show that $K_{\varepsilon, \sigma}^{(n l)}$ vanishes under the above four assumptions. From the Yang-Baxter equation (2.3) and the assumptions 1 and 3 , we obtain

$$
K_{\varepsilon, \sigma}^{(n l)}\left(\zeta^{\prime} \mid \zeta_{N-1}\right)^{[++]}=0
$$

The intertwining property of the $S$ matrix implies

$$
S_{N-1 \mid 1, \cdots, N-2}\left(\zeta_{N-1} \mid \zeta^{\prime}\right)\left(\pi_{\zeta^{\prime}} \otimes \pi_{\zeta_{N-1}}\right) \circ \Delta^{\prime}(y)=\left(\pi_{\zeta^{\prime}} \otimes \pi_{\zeta_{N-1}}\right) \circ \Delta(y) S_{N-1 \mid 1, \cdots, N-2}\left(\zeta_{N-1} \mid \zeta^{\prime}\right), \quad y \in U .
$$

Therefore noting $G_{\varepsilon}^{(n-1 l-1)} \in V^{(n-1 l-l)}$ we find from the assumption 4

$$
\pi_{\left(\zeta^{\prime}, \zeta_{N-1},-\sigma \zeta_{N-1} \tau\right)}\left(f_{0}\right) K_{\varepsilon, \sigma}^{(n l)}\left(\zeta^{\prime} \mid \zeta_{N-1}\right)=0 .
$$

Equations (4.1) and (4.2) imply that $K_{\varepsilon, \sigma}^{(n l)}$ has the following form

$$
K_{\varepsilon, \sigma}^{(n l)}\left(\zeta^{\prime} \mid \zeta_{N-1}\right)=K_{\varepsilon, \sigma}^{\prime(n l)}\left(\zeta^{\prime} \mid \zeta_{N-1}\right) \otimes u_{\sigma}
$$

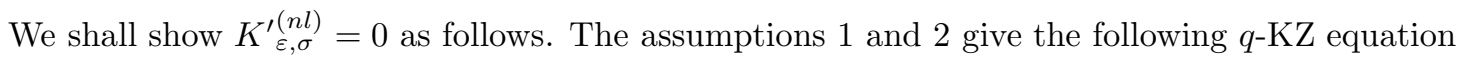

$$
P_{N-1 N} G_{\varepsilon}^{(n l)}\left(\zeta^{\prime}, \zeta_{N-1}, \zeta_{N}\right)=r_{\varepsilon}^{(l-n)}\left(\zeta_{N} q^{2}\right) D_{N-1}^{(l-n)} S_{1, \cdots, N-2 \mid N-1}\left(\zeta^{\prime} \mid \zeta_{N} q^{2}\right) G_{\varepsilon}^{(n l)}\left(\zeta^{\prime}, \zeta_{N} q^{2}, \zeta_{N-1}\right) .
$$

Taking the residue at $\zeta_{N}=-\sigma \zeta_{N-1} \tau$, we obtain

$$
P_{N-1 N} A_{\varepsilon, \sigma}^{(n l)}\left(\zeta^{\prime} \mid-\sigma \zeta_{N-1} \tau\right)=-r_{\varepsilon}^{(l-n)}\left(\zeta_{N-1}\right) D_{N-1}^{(l-n)} S_{1, \cdots, N-2 \mid N-1}\left(\zeta^{\prime} \mid \zeta_{N-1}\right) A_{\varepsilon, \sigma}^{(n l)}\left(\zeta^{\prime} \mid \zeta_{N-1}\right) .
$$


From the unitarity and crossing symmetry of the $S$ matrix (2.5), (2.6), and the condition for $r^{(k)}(\zeta)$ (2.10), it follows that $B_{\varepsilon, \sigma}^{(n l)}\left(\zeta^{\prime} \mid \zeta_{N-1}\right)$ also satisfies the above equation. From these we find

$$
\sigma K_{\varepsilon, \sigma}^{\prime(n l)}\left(\zeta^{\prime} \mid-\sigma \zeta_{N-1} \tau\right) \otimes u_{\sigma}=-r_{\varepsilon}^{(l-n)}\left(\zeta_{N-1}\right) D_{N-1}^{(l-n)} S_{1, \cdots, N-2 \mid N-1}\left(\zeta^{\prime} \mid \zeta_{N-1}\right) K_{\varepsilon, \sigma}^{\prime(n l)}\left(\zeta^{\prime} \mid \zeta_{N-1}\right) \otimes u_{\sigma} .
$$

By considering the difference of the $[-+]$ component and $\sigma \times$ the $[+-]$ component of the above equation, we obtain

$$
M^{(n l)}\left(\zeta^{\prime} \mid \zeta_{N-1}\right) K_{\varepsilon, \sigma}^{\prime(n l)}\left(\zeta^{\prime} \mid \zeta_{N-1}\right)=0
$$

Here

$$
M^{(n l)}\left(\zeta^{\prime} \mid \zeta_{N-1}\right)=\operatorname{tr}_{N-1}\left(\bar{D}_{N-1}^{(l-n)} R_{1, \cdots, N-2 \mid N-1}\left(\zeta^{\prime} \mid \zeta_{N-1}\right)\right) \in \operatorname{End}\left(V^{\otimes N-2}\right), \quad \bar{D}^{(k)}=\left(\begin{array}{cc}
1 & 0 \\
0 & -1
\end{array}\right) D^{(k)}
$$

and $\operatorname{tr}_{N-1}$ signifies the trace on the $(N-1)$-th space. Since the matrix $M^{(n l)}$ is invertible for generic $\zeta_{j}$ (for example, consider the special case $\left.\zeta_{1}=\cdots=\zeta_{N-1}\right)$, we obtain $K_{\varepsilon, \sigma}^{(n l)}\left(\zeta^{\prime} \mid \zeta_{N-1}\right)=0$.

We have already shown that the function family $G_{\varepsilon}^{(n l)}(\zeta)$ defined in section 2 satisfies the assumptions $1-3$ of Proposition 4.1. Therefore Theorem 2.1 is proved if we show the following lemma.

\section{Lemma 4.2}

$$
\left(\pi_{\left(\zeta_{1}, \cdots, \zeta_{N}\right)}\left(f_{0}\right)\right)\left\langle\Delta^{(n l)}\right\rangle\left(x_{1}, \cdots, x_{m} \mid \zeta_{1}, \cdots, \zeta_{N}\right)=0
$$

Proof This lemma is proved similarly as Lemma 3 in Chap.7 in [幽. Set

$$
\begin{aligned}
& P^{(n-1 l+1)}\left(x_{1}, \cdots, x_{m}\left|z_{1}, \cdots, z_{n-1}\right| z_{n}, \cdots, z_{N}\right) \\
= & (-\tau)^{l} \prod_{j=1}^{n-1} \zeta_{j}^{-1} \prod_{i=n}^{N} \prod_{j=1}^{n-1}\left(z_{i}-z_{j} \tau^{2}\right)\left(\pi_{\left(\zeta_{1}, \cdots, \zeta_{N}\right)}\left(f_{0}\right)\left\langle\Delta^{(n l)}\right\rangle\left(x_{1}, \cdots, x_{m} \mid \zeta_{1}, \cdots, \zeta_{N}\right)\right)^{-\cdots-+\cdots+} .
\end{aligned}
$$

Then thanks to the $R$ symmetry we obtain

$$
\begin{aligned}
& P^{(n-1 l+1)}\left(x_{1}, \cdots, x_{m}\left|z_{1}, \cdots, z_{n-1}\right| z_{n}, \cdots, z_{N}\right) \\
= & \sum_{k=n}^{N} \frac{\prod_{j=1}^{n-1}\left(z_{k}-z_{j} \tau^{2}\right)}{\prod_{\substack{i=n \\
i \neq k}}^{N}\left(z_{k}-z_{i}\right)} \Delta^{(n l)}\left(x_{1}, \cdots, x_{m}\left|z_{1}, \cdots, z_{n-1}, z_{k}\right| z_{n}, \stackrel{k}{\wedge}, z_{N}\right) .
\end{aligned}
$$

From the recurrence relation of $\Delta^{(n l)}$ [1], we can see that $P^{(n-1 l+1)}$ satisfies

$$
P^{(n-1 l+1)}\left(x_{1}, \cdots, x_{m}\left|z^{\prime}, a\right| z^{\prime \prime}, a \tau^{2}\right)=\prod_{\mu=1}^{m}\left(x_{\mu}-a \tau\right) \sum_{\nu=1}^{m}(-1)^{m+\nu} h^{(N-2)}\left(x_{\nu} \mid z^{\prime}, z^{\prime \prime}\right) P^{(n-2 l)}\left(x_{1}, \stackrel{\nu}{\nu} \cdot, x_{m}\left|z^{\prime}\right| z^{\prime \prime}\right)
$$

where $z^{\prime}=\left(z_{1}, \cdots, z_{n-2}\right)$ and $z^{\prime \prime}=\left(z_{n}, \cdots, z_{N-1}\right)$. We further find from the properties of $\Delta^{(n l)}$ as a polynomial [1] that $P^{(n-1 l+1)}$ is a homogeneous polynomial of degree $\left(\begin{array}{c}m \\ 2\end{array}\right)+(n-1) l-1$, antisymmetric with respect to $x_{\mu}$ 's and symmetric with respect to $\left\{z_{1}, \cdots, z_{n-1}\right\}$ and $\left\{z_{n}, \cdots, z_{N}\right\}$, respectively. Moreover from the power counting $P^{(0 l+1)}=0$. From these properties we can show $P^{(n-1 l+1)}=0$. 


\section{Discussion}

In this section we discuss the function family $G_{\varepsilon}^{(n l)}(\zeta)$ satisfying the three axioms 2.72 .9 ) in the context of the $X X Z$ model in the ferroelectric regime $(-1<q<0)$. In refs [6. [7], this model was solved by utilizing the representation theory of $U_{q}\left(\widehat{\mathfrak{s l}}_{2}\right)$. Firstly following mainly their notations, we summarize the necessary results on this model. See those references for further details. Let $V\left(\Lambda_{i}\right)(i=0,1)$ be the level 1 highest weight module of $U_{q}\left(\widehat{\mathfrak{s l}}_{2}\right)$ and set $\mathcal{H}=V\left(\Lambda_{0}\right) \oplus V\left(\Lambda_{1}\right)$ and $\mathcal{F}^{(i j)}=V\left(\Lambda_{i}\right) \otimes V\left(\Lambda_{j}\right)^{* b}$. Here the superscript $* b$ signifies the dual module regarded as a left module by some anti-automorphism $b$. Then the space $\mathcal{F}$ on which the $X X Z$ hamiltonian acts is identified with

$$
\mathcal{F}=\mathcal{H} \otimes \mathcal{H}^{* b}=\bigoplus_{i, j=0,1} \mathcal{F}^{(i j)}
$$

In this space $\mathcal{F}$ there exist two ground states of the hamiltonian, which belong to $\mathcal{F}^{(00)}$ and $\mathcal{F}^{(11)}$. We denote them and their dual vectors by $|\mathrm{vac}\rangle_{(i)}$ and ${ }_{(i)}\langle\mathrm{vac}|(i=0,1)$, respectively. The creation and annihilation operators $\varphi_{\varepsilon}^{*}(\zeta), \varphi^{\varepsilon}(\zeta) \in \oplus_{i j} \operatorname{Hom}\left(\mathcal{F}^{(i j)}, \mathcal{F}^{(1-i j)}\right)(\varepsilon= \pm,|\zeta|=1)$ that diagonalize the hamiltonian can be constructed in terms of the vertex operators of the algebra. They have the following property

$$
\varphi^{\varepsilon}(\zeta)|\operatorname{vac}\rangle_{(i)}=0, \quad{ }_{(i)}\langle\operatorname{vac}| \varphi_{\varepsilon}^{*}(\zeta)=0,
$$

and the whole space $\mathcal{F}$ and its dual space $\mathcal{F}^{*}$ are spanned by the vectors

$$
\varphi_{\varepsilon_{1}}^{*}\left(\zeta_{1}\right) \cdots \varphi_{\varepsilon_{N}}^{*}\left(\zeta_{N}\right)|\operatorname{vac}\rangle_{(i)} \quad \text { and } \quad{ }_{(i)}\langle\operatorname{vac}| \varphi^{\varepsilon_{1}}\left(\zeta_{1}\right) \cdots \varphi^{\varepsilon_{N}}\left(\zeta_{N}\right) \quad(i=0,1, N=0,1, \cdots)
$$

respectively. These operators further satisfy the following property

$$
\varphi_{\varepsilon}^{*}(-\zeta)=(-1)^{\left(\varepsilon-(-1)^{i}\right) / 2} \varphi_{\varepsilon}^{*}(\zeta), \quad \varphi^{\varepsilon}(-\zeta)=(-1)^{\left(\varepsilon+(-1)^{i}\right) / 2} \varphi^{\varepsilon}(\zeta) \quad \text { on } \mathcal{F}^{(i j)} \quad(i, j=0,1)
$$

and the commutation relations

$$
\begin{aligned}
& P_{12} \varphi^{V}\left(\zeta_{1}\right) \varphi^{V}\left(\zeta_{2}\right)=S_{12}\left(\zeta_{1} / \zeta_{2}\right) \varphi^{V}\left(\zeta_{2}\right) \varphi^{V}\left(\zeta_{1}\right), \quad P_{12} \varphi^{* V}\left(\zeta_{1}\right) \varphi^{* V}\left(\zeta_{2}\right)=S_{12}\left(\zeta_{1} / \zeta_{2}\right) \varphi^{* V}\left(\zeta_{2}\right) \varphi^{* V}\left(\zeta_{1}\right), \\
& P_{12} \varphi^{V}\left(\zeta_{1}\right) \varphi^{* V}\left(\zeta_{2}\right)=S_{12}\left(-\zeta_{1} / q \zeta_{2}\right) \varphi^{* V}\left(\zeta_{2}\right) \varphi^{V}\left(\zeta_{1}\right)+\frac{1}{2} \sum_{\sigma= \pm} \sigma \delta\left(\sigma \zeta_{1} / \zeta_{2}\right) u_{\sigma} \otimes\left(P_{0}+\sigma P_{1}\right) .
\end{aligned}
$$

In the above equations,

$$
\varphi^{* V}(\zeta)=\sum_{\varepsilon} v_{\varepsilon} \otimes \varphi_{-\varepsilon}^{*}(\zeta), \quad \varphi^{V}(\zeta)=\sum_{\varepsilon} v_{\varepsilon} \otimes \varphi^{\varepsilon}(\zeta) \in \bigoplus_{i j} \operatorname{Hom}\left(\mathcal{F}^{(i j)}, V \otimes \mathcal{F}^{(1-i j)}\right)
$$

$P_{i}$ is the projection operator to the subspace $\oplus_{j=0,1} \mathcal{F}^{(i j)}$ and $\delta(\zeta)=\sum_{m \in \mathbb{Z}} \zeta^{m}$. Note that we consider the creation and annihilation operators in the principal picture. (See, for example, eqs. $(2.2,4.8-9)$ in [1] for the above properties of these operators in this picture.)

Now we shall discuss the form factors of this model. For simplicity, we shall consider an operator $\mathcal{O} \in$ $\operatorname{End}(\mathcal{F})$ that has the form $i d \otimes O\left(O \in \operatorname{End}\left(\mathcal{H}^{*}\right)\right)$ and satisfies

$$
\left(i d \otimes \bar{\Psi}^{V}(\zeta)\right) \mathcal{O}=r(-\zeta q) D \mathcal{O}\left(i d \otimes \bar{\Psi}^{V}(\zeta)\right) .
$$


Here $r(\zeta)$ is a scalar function, $D$ is a diagonal matrix acting on the space $V$ and $\bar{\Psi}^{V}(\zeta): \mathcal{H}^{* b} \rightarrow \mathcal{H}^{* b} \otimes V_{\zeta}$ is the type II vertex operator in the terminology of [6, 7]. We denoted by $V_{\zeta}$ the vector representation defined in 2.1). For such $\mathcal{O}$ we introduce the form factor by

$$
G_{\varepsilon}^{(N)}\left(\zeta_{1}, \cdots, \zeta_{N}\right)=\sum_{i=0,1} \varepsilon^{i} \times{ }_{(i)}\left\langle\operatorname{vac}\left|\mathcal{O} \varphi^{* V}\left(\zeta_{N}\right) \cdots \varphi^{* V}\left(\zeta_{1}\right)\right| \operatorname{vac}\right\rangle_{(i+N)} \quad(\varepsilon= \pm 1) .
$$

The commutation relations among the creation operators imply the first axiom. From (5.1), we have

$$
G_{\varepsilon}^{(N)}\left(\zeta_{1}, \cdots,-\zeta_{j}, \cdots, \zeta_{N}\right)^{-\cdots-+\cdots+}=(-1)^{N-j+H(n \geq j)} G_{-\varepsilon}^{(N)}\left(\zeta_{1}, \cdots, \zeta_{j}, \cdots, \zeta_{N}\right)^{-\cdots-+\cdots+},
$$

where $H$ is the step function and $n$ is the number of the superscript - of $G_{\varepsilon}^{(N)}$. Note that for $G_{\varepsilon}^{(n l)}(\zeta)$ defined in section 2 the condition $(5.3)$ is equivalent to

$$
\vartheta_{\varepsilon}^{(n l)}\left(x_{1}, \cdots, x_{m} \mid \zeta_{1}, \cdots,-\zeta_{j}, \cdots, \zeta_{N}\right)=\vartheta_{-\varepsilon}^{(n l)}\left(x_{1}, \cdots, x_{m} \mid \zeta_{1}, \cdots, \zeta_{j}, \cdots, \zeta_{N}\right) .
$$

Let $\zeta^{ \pm}$signify $(1 \pm \eta) \zeta(0<\eta \ll 1)$ for $\zeta$, and $\zeta^{\prime \prime}$ be the abbreviation $\left(\zeta_{1}, \cdots, \zeta_{N-1}\right)$. Set $r_{\varepsilon}(\zeta)=\varepsilon r(\zeta)$ as before and set further

$$
G_{\varepsilon, \sigma, k}^{(N-2)}\left(\zeta_{1}, \cdots, \zeta_{N-1}\right)=P_{k, k+1} \cdots P_{N-2, N-1} G_{\sigma \varepsilon}^{(N-2)}\left(\zeta_{1}, \stackrel{k}{\wedge}, \zeta_{N-1}\right) \otimes u_{\sigma} .
$$

Then similarly as in [1], thanks to the condition (5.2) the following matrix element of $\mathcal{O}$ can be expressed in terms of the form factors in two different ways:

$$
\begin{aligned}
& \sum_{i=0,1} \varepsilon^{i} \times{ }_{(i)}\left\langle\operatorname{vac}\left|\varphi^{V}\left(\zeta_{N}\right) \mathcal{O} \varphi^{* V}\left(\zeta_{N-1}\right) \cdots \varphi^{* V}\left(\zeta_{1}\right)\right| \operatorname{vac}\right\rangle_{(i+N)} \\
= & G_{\varepsilon}^{(N)}\left(\zeta^{\prime \prime}, \zeta_{N}^{-} /(-q)\right)+\frac{1}{2} \sum_{\substack{1 \leq k \leq N-1 \\
\sigma= \pm}} \delta\left(\sigma \zeta_{k} / \zeta_{N}\right) S_{k+1, \cdots, N-1 \mid k}\left(\zeta_{k+1}, \cdots, \zeta_{N-1} \mid \zeta_{k}\right) G_{\varepsilon, \sigma, k}^{(N-2)}\left(\zeta^{\prime \prime}\right) \\
= & r_{\varepsilon}\left(-\zeta_{N} q\right) D_{N}\left(P_{N-1, N} \cdots P_{1,2} G_{\varepsilon}^{(N)}\left(-q \zeta_{N}^{+}, \zeta^{\prime \prime}\right)\right. \\
& \left.\quad+\frac{1}{2} \sum_{\substack{1 \leq k \leq N-1 \\
\sigma= \pm}} \sigma^{N+1} \delta\left(\sigma \zeta_{k} / \zeta_{N}\right) S_{k \mid 1, \cdots, k-1}\left(\zeta_{k} \mid \zeta_{1}, \cdots, \zeta_{k-1}\right) G_{\varepsilon, \sigma, k}^{(N-2)}\left(\zeta^{\prime \prime}\right)\right) .
\end{aligned}
$$

The last equality of (5.5) implies that $G_{\varepsilon}^{(N)}(\zeta)$ satisfies also the second and third axioms. Conversely, suppose that we are given the $G_{\varepsilon}^{(n l)}(\zeta)$ satisfying the three axioms and (5.3). Then we can define an operator $\mathcal{O} \in$ $\operatorname{End}(\mathcal{F})$ by giving the matrix elements in terms of $G_{\varepsilon}^{(n l)}(\zeta)$ in two different ways as in the original work 四. The second and third axioms assures the equivalence of the two expressions. (Equation (5.5) shows one simplest example.) These two expressions enable us to calculate the commutation relations of the thus defined operators, though the knowledge of the poles other than annihilation poles of the $G_{\varepsilon}^{(n l)}(\zeta)$ is necessary. The classification of all solutions to the conditions 2.15 2.17, 5.4) and the identification of them with $\mathcal{O} \in \operatorname{End}(\mathcal{F})$ are still open questions.

Finally we shall give an example of $\vartheta_{\varepsilon}^{(n l)}$ in the case

$$
r_{\varepsilon}^{(n l)}(\zeta)=\varepsilon \prod_{k=1}^{N-2 m} t\left(\zeta / \xi_{k}\right), \quad t(\zeta)=\zeta^{-1} \frac{\theta\left(q z \mid q^{4}\right)}{\theta\left(q z^{-1} \mid q^{4}\right)},
$$


where $m=n-1$ for $n=l$, $=n$ for $n<l$ as before and $\xi_{k} \in \mathbb{C}$. In this case one solution to the conditions (2.15 $2.17,5.4)$ is given by

$$
\frac{\vartheta_{\varepsilon}^{(n l)}\left(x_{1}, \cdots, x_{m} \mid \zeta_{1}, \cdots, \zeta_{N}\right)}{\prod_{j=1}^{N} \zeta_{j}^{N-2 m} \prod_{1 \leq \mu<\nu \leq m}\left(x_{\mu} \theta\left(x_{\nu} / x_{\mu} \mid q^{2}\right)\right)}=\text { const } \varepsilon^{N} \theta\left(-\varepsilon u \mid q^{2}\right) \prod_{\substack{1 \leq k \leq N-2 m \\ 1 \leq \mu \leq m}} \theta\left(\xi_{k}^{2} / x_{\mu} \mid q^{4}\right) \prod_{\substack{1 \leq j \leq N \\ 1 \leq k \leq N-2 m}} f\left(z_{j} / \xi_{k}^{2}\right),
$$

where

$$
u=(-1)^{N-m} \tau^{N / 2-3 m} \frac{\prod_{\mu=1}^{m} x_{\mu} \prod_{k=1}^{N-2 m} \xi_{k}}{\prod_{j=1}^{N} \zeta_{j}}, \quad f(z)=\frac{\left(q^{5} z ; q^{4}, q^{4}\right)_{\infty}\left(q^{5} / z ; q^{4}, q^{4}\right)_{\infty}}{\left(q^{3} z ; q^{4}, q^{4}\right)_{\infty}\left(q^{3} / z ; q^{4}, q^{4}\right)_{\infty}} .
$$

\section{Acknowledgments}

The authors would like to thank M. Jimbo and T. Miwa for the earlier collaborations. Y.-H.Q. is supported by the Australian Research Council.

\section{References}

[1] Jimbo, M., Kojima, T., Miwa, T. and Quano, Y.-H., 1994: Smirnov's integrals and quantum KnizhnikZamolodchikov equation of level 0, J. Phys. A27 3267-3283.

[2] Frenkel, I. B. and Reshetikhin, N. Yu., 1992: Quantum affine algebras and holonomic difference equations, Comm. Math. Phys. 146 1-60.

[3] Kojima, T. and Quano, Y.-H., 1994: Quantum Knizhnik-Zamolodchikov equation for $U_{q}\left(\widehat{\mathfrak{s l}}_{n}\right)$ and integral formula, J. Phys. A27 6807-6826.

[4] Smirnov, F. A., 1992: Form Factors in Completely Integrable Models of Quantum Field Theory, World Scientific, Singapore.

[5] Smirnov, F. A., 1992: Dynamical symmetries of massive integrable models 1,2: Int. J. Mod. Phys. 7A, Suppl. 1B 813-837, 839-858.

[6] Davies, B., Foda, O., Jimbo, M., Miwa, T. and Nakayashiki, A., 1993: Diagonalization of the $X X Z$ Hamiltonian by Vertex Operators, Comm. Math. Phys. 151 89-153.

[7] Jimbo, M. and Miwa, T. , 1994: Algebraic analysis of solvable lattice models, CBMS Regional Conferences Series in Mathematics.

[8] Jimbo, M., Miki, K., Miwa, T. and Nakayashiki, A., 1992: Correlation functions of the $X X Z$ model for $\Delta<-1$, Phys. Lett. 168A 256-263.

[9] Konno, H., 1994: Free field representation of quantum affine algebra $U_{q}\left(\widehat{\mathfrak{s l}_{2}}\right)$ and form factors in higher spin $X X Z$ model, preprint YITP/K-1074. 
[10] Pakuliak, S., 1993: Annihilation poles for form factors in $X X Z$ model, preprint RIMS-933.

[11] Miki, K., 1994: Creation/annihilation operators and form factors of the $X X Z$ model, Phys. Lett. 186A $217-224$.

[12] See e.g., Jimbo, M., 1992: Topics from Representations of $U_{q}(\mathfrak{g})-$ An Introductory Guide to Physicists, Nankai Lectures on Mathematical Physics, World Scientific, Singapore, pp. 1-61. 